\title{
PHYSICO-CHEMICAL CHARACTERISTICS OF WHITE SUGAR FRACTIONS SEPARATED BY CRYSTAL SIZES
}

${ }^{1}$ N. Maravić, ${ }^{1} Z$. Šereš, ${ }^{1}$ J. Petrović, ${ }^{1}$ L. Dokić, ${ }^{1}$ D. Š. Simović, ${ }^{1}$ M. Djordjević, ${ }^{1}$ M. Djordjević, ${ }^{1}$ I. Nikolić

${ }^{1}$ Department of Carbohydrate Food Engineering, Faculty of Technology, University of Novi Sad, 21000, Bul. Cara Lazara 1, Novi Sad, Serbia

e-mail: maravic@tf.uns.ac.rs, zitas@tf.uns.ac.rs, jovana@tf.uns.ac.rs, ldbaucal@tt.uns.ac.rs, dragana@tf.uns.ac.rs, miljanadj@tf.uns.ac.rs,maridjo@tf.uns.ac.rs, ivananikolic@tf.uns.ac.rs

\begin{abstract}
According to the EU Council Directive 2001/111/EC, related to certain sugars intended for human consumption, a great attention is paid to color in sugar. On the other hand, the non-sucrose compounds having intensive color tend to build into the sucrose crystals. Since Serbia has to work on the new rules concerned sugar quality and harmonization with EU standards, some investigations on sugar crystals are carried out at the request of some sugar factories. Investigations are carried out on sugar crystal size dependence on sugar color and on dissolution rate of different sugar crystal size fractions with the aim to create new sugar products. In this study, sugar samples with different sugar color are divided into 5 fractions by crystal size, defining size distributions. In each fraction the color in solution and the type of color are measured, as well as other relevant physico-chemical parameters. The conclusion is that the sugar color type depends on the crystal size, but sugar crystal solution is not dependent on crystal size except a fraction smaller than $200 \mu \mathrm{m}$, which has $30-75 \%$ higher sugar color in solution than the other crystal size fractions.
\end{abstract}

Keywords: sugar crystal, crystal size, sensory analysis, dissolution rate.

\section{INTRODUCTION}

World sugar production (from beet and cane) in 2005 was 149.5 million metric tons and consumption was 150.5 metric tons. World sugar consumption has grown by $3 \%$ annually since 1985 , and the world production has kept up with this increase in demand for sugar. In recent years, world sugar consumption has increased almost yearly, to about $25 \mathrm{~kg}$ per capita per year in 2005[1].

Sugar manufacturers in different countries produce sugar of specific crystal sizes, primary depending on the habits of the household and commercial use. Therefore, sugar factories in USA prefer the production of fine sugar, those in Europe prefer medium-size sugar, and those in Asia, Africa, and the Far East prefer coarse sugar [2].

In most countries, fine and medium-size sugars are usually the standard, and extra-fine, coarse, and extracoarse sugars are considered specialty sugars for which customers pay a premium. The numerous advantages of extra-fine sugar, mostly due to its high surface area, enable shorter mixing and milling processes in baking and chocolate industry [1]. Special-size sugars are usually produced from fractioning the sugar by passing it through a multiple-screening operation [3].

Moreover, sugar producers have to fulfil strict targets for sugar color in order to meet consumer's demands. Therefore, the special interest in sugar production is dedicated to the purification of juice extracted from the crop plant in order to obtain sugar crystal with minimum content of colorants [4].

During the process of sugar production, colored matter are easily adsorbed to the sucrose crystal surface or incorporated into the sugar crystal and thus increase the color of the sugar solution and lower the quality of the final product. The coloring substances occurred in the sugar manufacturing process can be classified as: caramel, melanins, melanoidins, products of the sucrose decomposition and polyphenol ion complexes [3]. 
Considering the existing literature information on the different ways of incorporation of certain types of colored matter in sugar crystals, there is a reasonable basis for assuming that the color of various crystal sizes from the same sugar sample differs.

The aim of this paper is to investigate the effect of sugar crystal size of four fractions from the same sugar sample on the determination of sugar color. Furthermore, the analysis of sensory properties and physicochemical characteristics of different size sugar crystals is presented and discussed.

\section{MATERIALS AND METHODS}

The samples of the white sugar crystals were obtained from the sugar factory "Crvenka", Serbia. The white sugar crystals were divided into fractions using the laboratory sieve. As presented in Table 1, 4 crystal fractions were obtained. The fraction with crystal sizes under $200 \mu \mathrm{m}$ was classified as sugar powder and therefore was not used in the further investigation. In the further analysis dissolution rate of obtained fractions is calculated. For the purpose of this experiment distilled water was used as a solvent. Distilled water temperature was kept constant at $20^{\circ} \mathrm{C}$ and controled using the thermostat (VELP ${ }^{\circledR}$ Scientifica, Italy). The mixing of the solution was conducted using the automatic mixer at a constant rotating speed of $100 \mathrm{rpm}$. Under these conditions, after the addition of sugar crystal fraction, solution was sampled on a 10s period using the micropippete. Disolved sugar was measured using the refractometer (Carl Zeiss, Germany) and dissolution rate was calculated using the obtained results of sugar content in the sample.

Furthermore, in each fraction, the color in solution is measured. Determination of the solution color of white sugar is conducted in accordance to the ICUMSA (The International Commission for Uniform Methods of Sugar Analysis) Methods GS 1/3-7, GS 2/3-10 and GS 2/3-10. According to the ICUMSA, absorbance of white sugar solution is measured using the laboratory spectrophotometer (SPECOL 220-MA 9522) at a wavelength of $420 \mathrm{~nm}$ and cuvettes with optical path-length of $5 \mathrm{~cm}$. ICUMSA color is calculated as follows (1):

$$
\text { ICUMSA Color }(I U)=\frac{1000 \times A}{l \times c}
$$

Where $A=$ absorbency of the solution,

$1=$ the optical path-length,

$\mathrm{c}=$ solution concentration $(\mathrm{g} / \mathrm{mL})$.

The sensory properties of the sugar crystal fractions were determined in the further research. Sensory analysises is performed according to the ISO standards, adapted to the evaluation of examined white sugar crystals (ISO 4121, 2003; ISO 8589, 2007; ISO 8586-2, 2008). The investigated parameters relate to the texture and taste including: granulation, sweetness, mouth melting properties, crystal sharpness and general admissibility. The assessment of these parameters is performed using the numerical scale with seven levels of assessment for each quality parameter.

\section{RESULTS}

Firstly, dissolution rate of the sugar fractions will be discussed. The results obtained from the experimental determination of dissolution rate are presented in Tab. 1. Moreover, in order to make a clear comparison 
between the inspected fractions, the results from Tab. 1 are illustrated in Fig. 1. As expected, the highest dissolution rate is observed in the experiments where smallest sugar crystals are used. Dissolution rate of the different sugar crystal sizes can be clearly distinguished after 20 s of the experiment, where it can be noticed that the Fraction $1(200 / 400 \mu \mathrm{m})$ has significantly higher dissolution rate, $30 \%$ and $50 \%$, comparing to the larger crystal fractions $2(400 / 600 \mu \mathrm{m})$ and fraction $3(600 / 800 \mu \mathrm{m})$, respectively. During the time of the experiment, the solubility of all factions increases at $20^{\circ} \mathrm{C}$, however with different intensity.

Table 1. Solubility of a different sugar fractions over time

\begin{tabular}{|c|c|c|c|c|}
\hline & \multicolumn{4}{|c|}{ Solubility (\%) } \\
\hline Range of crystal sizes $(\mu \mathrm{m})$ & $200 / 400$ & $400 / 600$ & $600 / 800$ & $>800$ \\
\hline time $(\mathrm{s})$ & Fraction 1 & Fraction 2 & Fraction 3 & Fraction 4 \\
\hline 0 & 0 & 0 & 0 & 0 \\
\hline 10 & 10.0 & 12.5 & 5.0 & 2.5 \\
\hline 20 & 25.0 & 17.5 & 12.5 & 10.0 \\
\hline 30 & 42.5 & 22.5 & 20.0 & 15.0 \\
\hline 40 & 47.5 & 30.0 & 22.5 & 20.0 \\
\hline 50 & 50.0 & 32.5 & 30.0 & 22.5 \\
\hline 60 & 52.5 & 35.0 & 32.5 & 27.5 \\
\hline
\end{tabular}

The most intensive increase in dissolution rate is observed again in the fraction $200 / 400 \mu \mathrm{m}$, where the solubility increases linearly up to 30 seconds. The linear increase of dissolution rate continued after $30 \mathrm{~s}$ but with far less intensity.

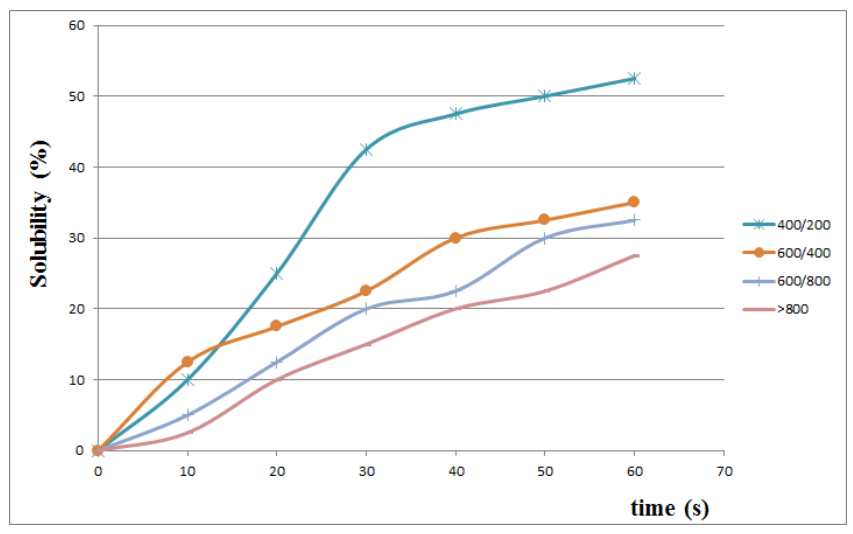

Figure 1. Solubility of sugar crystal fractions over time 
The linear increase of dissolution rate continued after $30 \mathrm{~s}$ but with far less intensity. This can be clearly observed in the Fig. 2, where the curve of dissolution rate vs. time is presented. The maximum dissolution rate regarding the smallest crystal fraction $(200 / 400 \mu \mathrm{m})$ with value of $0.14 \mathrm{~g} / \mathrm{s}$ is obtained after $30 \mathrm{~s}$ of the experiment. As it can be noticed from Figure 2, significantly lower values are obtained for larger sugar crystals.

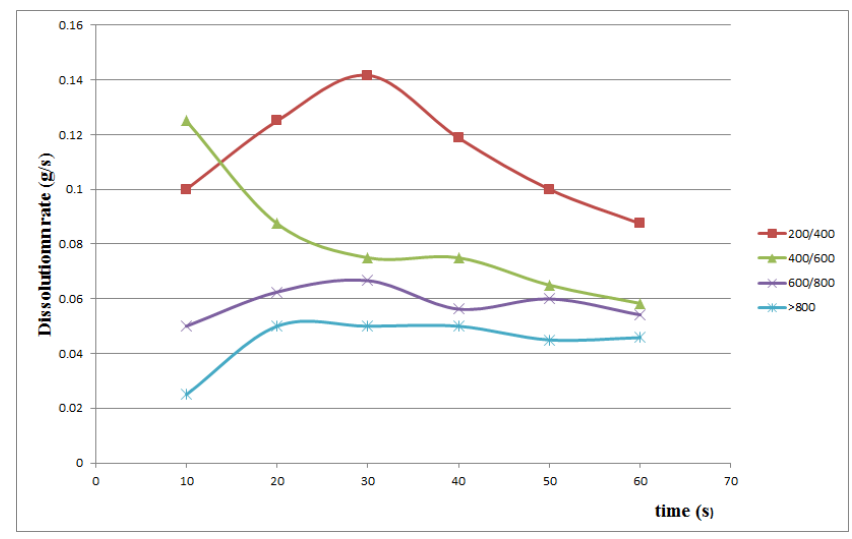

Figure 2. Dissolution rate vs. time curve for the investigates fractions

At the end of the experiment, after $60 \mathrm{~s}$, Fraction 1 solubility reaches $52 \%$, while all other fractions did not exceed $35 \%$. Therefore, from the curves shown in Fig. 1 it can be concluded that the Fraction 1 $(200 / 400 \mu \mathrm{m})$ of crystal sugar dissolves fastest compared to other fractions of crystal sugar.

The results of the sensory analysis of different crystal sugar granulation are given in Tab. 2 and graphically shown in Fig 3 using the QDA (Quality Descriptive Analysis) diagram. For the purpose of this experiment 5 different parameters are assessed. The parameters are assessed in the following plan range: granulation from extremely small to extremely large; mouth melting from extremely slow melting to extremely fast melting; sweetness from pronouncedly weak sweetness to extremely sweet; crystal sharpness from completely smooth to extremely sharp; general admissibility from not appropriate to completely appropriate. The numeric range between the two extremes is given from 1 to 7 points. 
Table 2. Average ratings of sensory analysis of investigated parameters grouped by different sugar fractions

\begin{tabular}{|l|c|c|c|c|}
\hline \multicolumn{1}{|c|}{ Parameter } & $\begin{array}{c}\text { Fraction 1 } \\
200 / 400 \mu \mathrm{m}\end{array}$ & $\begin{array}{c}\text { Fraction 2 } \\
400 / 600 \mu \mathrm{m}\end{array}$ & $\begin{array}{c}\text { Fraction 3 } \\
600 / 800 \mu \mathrm{m}\end{array}$ & $\begin{array}{c}\text { Fraction 4 } \\
>800 \mu \mathrm{m}\end{array}$ \\
\hline Granulation & 2.5 & 3.8 & 5.8 & 6.9 \\
\hline Mouth melting & 6.8 & 6.1 & 3.3 & 105 \\
\hline Sweetness & 5.8 & 3.7 & 4.5 & 3.7 \\
\hline Crystal sharpness & 3.4 & 4.1 & 6.2 & 7.0 \\
\hline General admissibility & 6.8 & 6.8 & 4.5 & 2.0 \\
\hline
\end{tabular}

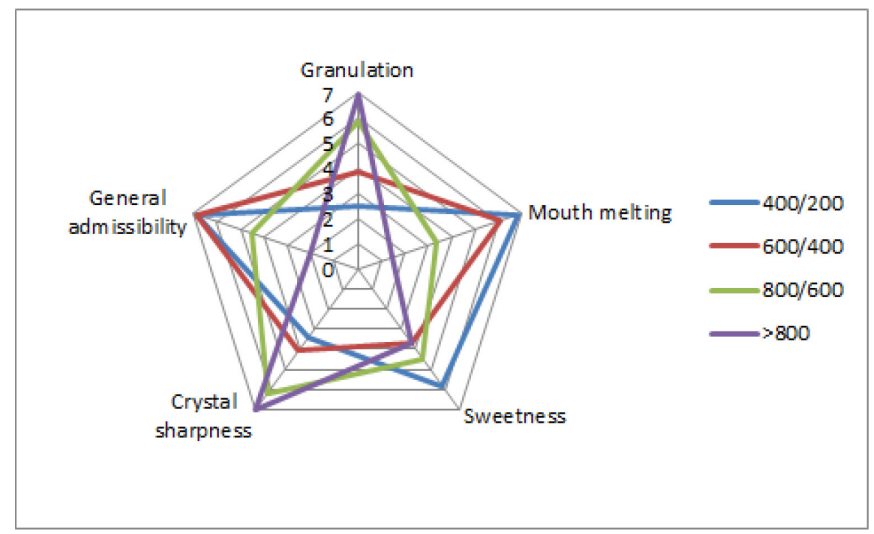

Figure 3. QDA diagram of the sensory evaluation relating to different sugar fractions

After the evaluation of the obtained ratings given by the professional assessors, the highest values for crystal sharpness are, as expected, given to the largest fractions $(600 / 800 \mu \mathrm{m}$ and $>800 \mu \mathrm{m})$ emphasizing their crystal size. The sugar fractions 1 and 2 are rated with 3.4 and 4.1, indicating optimal crystal sharpness and a pleasant mouth feeling.

As regards mouth melting properties, it is evident that the fraction $200 / 400 \mu \mathrm{m}$ has the best melting properties, confirmed by significantly high ratings (6.8), which is in accordance with the examination of the solubility in water of this fraction (Fig. 1 and Fig. 2).

However, Fraction 2 also obtained high rating concerning mouth melting properties with only 0.7 points less than Fraction 1. This can be explained by a high initial dissolution rate of this fraction confirmed in the first experiment (Fig. 2). The extremely low rating (1.5) concerning this characteristic is received for the sugar crystals above $800 \mu \mathrm{m}$ which is in accordance to the previous experiment.

The sweetness ratings are quite similar for the sugar crystals with sizes from 400 to $800 \mu \mathrm{m}$ and above, only the sugar crystals below $400 \mu \mathrm{m}$ received higher assessments (5.8), indicating extremely sweet taste induced by this sugar fraction. 
In the end, general admissibility of the all sugar fractions is assessed. Highest values are obtained for the sugar crystals from Fractions 1 and 2 (6.8) and the lowest value, indicating this fraction as not appropriate, is given to the Fraction 4 with the size of crystals above $800 \mu \mathrm{m}$.

Now, the results of the color measurements for each sugar fraction will be given and analyzed. The presented results (Tab. 3) indicate high dependence of solution color on specific sugar crystal size. It can be noticed that the solution color increases as the crystal size decrease. The largest sugar crystals had $35 \%$ lower solution color compared to the smallest sugar crystals. This phenomenon can be attributed to the greater surface area of the fractions with smaller sugar crystals. As the surface area increases more colored matter are able to contact the specific sugar crystal enabling adsorption or incorporation of these substances to the crystal. Considerably larger surface will be available to the adsorbent materials enhancing their possibility to permanently adsorb on a sucrose crystal. Moreover, the turbidity of the solution, which is in direct correlation with the absorbance of the unfiltered sample, (expressed as a difference between the color of unfiltered and filtered sugar solution) (Tab. 3) is significantly higher for the smaller crystal fractions. Higher turbidity is attributed to the specific larger number of crystals in the investigated solution resulting in a greater absorbency of the solution at $720 \mathrm{~nm}$.

Table 3. Solution color of the sugar sample

\begin{tabular}{|c|c|c|c|}
\hline $\begin{array}{c}\text { Crystal size } \\
(\mu \mathrm{m})\end{array}$ & Color (IU) & $\begin{array}{c}\text { Unfiltered } \\
\text { sample color } \\
(\mathrm{IU})\end{array}$ & $\begin{array}{c}\text { Turbidity of } \\
\text { sugar } \\
\text { solution }(\mathrm{IU})\end{array}$ \\
\hline $200 / 400$ & 62 & 324 & 262 \\
\hline $400 / 600$ & 50 & 184 & 134 \\
\hline $600 / 800$ & 43 & 102 & 59 \\
\hline$>800$ & 46 & 101 & 55 \\
\hline
\end{tabular}

\section{CONCLUSION}

The analysis of sensory properties and physico-chemical characteristics of different size sugar crystals is presented and discussed. On the basis of physical and chemical analysis it can be concluded that already starting from $10 \mathrm{~s}$ to $30 \mathrm{~s}$ of experiment about $50 \%$ more sugar of a fraction with smallest sugar crystals is dissolved compared to the fraction with larger sugar crystals, which is in accordance with the sensory assessment of sweetness and solubility. The maximum dissolution rate regarding the smallest crystal fraction $(200 / 400 \mu \mathrm{m})$ with value of $0.14 \mathrm{~g} / \mathrm{s}$ is obtained after $30 \mathrm{~s}$ of the experiment. Therefore, it can be concluded that due to the higher initial dissolution rate of corresponding sugar crystal fraction, sweetness sensing is proportionally more intense.

The general acceptability of sugar crystal fractions among evaluators is very interesting. The trained sensory assessors have opted for a fraction $200 / 400 \mu \mathrm{m}$ and $400 / 600 \mu \mathrm{m}$ with a score of 6.8 , with a description "completely appropriate" product, while the fraction $>800 \mu \mathrm{m}$ is assessed with 2 , ie. assessors find this product "not appropriate". 
ACKNOWLEDGMENT

The authors would like to thank "The Hellenic Sugar industry S.A.", Greece and "SUNOKO", Serbia and Ministry of Science and Technological Development of the Republic of Serbia (Project no. 31014) for donations.

\section{REFERENCES}

[1] M. Asadi, Beet-sugar handbook. John Wiley \& Sons, Inc, Hoboken, New Jersey, 2007

[2] P. W. Van der Poel, Sugar Technology Beet and Cane Sugar Manufacture PW van der Poel, H. Schiweck, T. Schwartz. Berlin: Verlag Dr. Bartens KG, pp. 479-563., 1998

[3] S. Šušić, S. Petrov, G. Kukić, V. Sinobad, P. Perunović, B. Koronsavac, D. Bašić, Osnovi tehnologije šećera, University of Belgrade, Belgrade, 1994

[4] N. Maravić, F. Kiss, L. Šereš, B. Bogdanović, B. Bogdanović, Z. Šereš, Economic analysis and LCA of an advanced industrial-scale raw sugar juice purification procedure. Food and Bioproducts Processing, 95, (2015), pp. 19-26. 